\title{
How to Use the FORTA ("Fit fOR The Aged") List to Improve Pharmacotherapy in the Elderly
}

\author{
Author \\ Affiliation \\ Key words \\ - polypharmacy \\ - elderly \\ - FORTA-list \\ - implicit tool \\ - positive list \\ negative list
}

\section{Wehling}

Department of Clinical Pharmacology, Center for Geriatric Pharmacology in Mannheim, Medical Faculty Mannheim, University of Heidelberg, Germany received 09.03 .2015

accepted 16.04.2015

\section{Bibliography \\ DOI http://dx.doi.org/ \\ 10.1055/s-0035-1549935 \\ Published online: \\ July 21, 2015 \\ Drug Res 2016; \\ 66: 57-62 \\ (c) Georg Thieme Verlag KG \\ Stuttgart · New York \\ ISSN 2194-9379}

\section{Correspondence}

\section{Prof. M. Wehling, MD}

Institute for Experimental and Clinical Pharmacology and Toxicology

Ruprecht-Karls-Universität Heidelberg,

Maybachstraße 14

D-68169 Mannheim

Germany

Tel.: + 49/621/3839631

Fax: +49/621/3839632

martin.wehling@medma.uni-

heidelberg.de

\section{Abstract}

$\nabla$

Background: Multimorbidity and polypharmacy are threats to elderly patients; improvement of medication is important and a novel listing approach (the FORTA list) should support this in clinical practice. Here we aim to describe procedural details of successful application of FORTA. FORTA labels range from A (indispensable), B (beneficial), C (questionable) to D (avoid), depending on evidence for safety, efficacy and overall age-appropriateness. As implicit tool it is only applicable if medical details of the patient are known; the drug selection process and secondary assessments are compiled into a manual for successful, embedded use of FORTA.

Discussion: A flow chart is developed for the complex process of medication in the elderly starting from history taking and diagnostic assessment including disease grading. This is

\section{Introduction and Background \\ $\nabla$}

Elderly multimorbid patients are likely to receive multiple drug treatments ( $\geq 5=$ polypharmacy); patients aged 65 and older take 5 or more drugs in $44 \%$ (male) and $57 \%$ (female) of cases and 10 or more drugs in $12 \%$ of cases [1]. Often aggravated by age-related alterations in pharmacokinetics and pharmacodynamics [2], polypharmacy may precipitate adverse drug reactions [3], which may in turn trigger the so-called prescribing cascade [4], drug-drug or drug-disease interactions, dosing and medication errors and even death [5]. It is estimated that approximately 2.1 million sideeffect-related hospital admissions and 100000 deaths occur per year in the U.S. [6].

Strategies for the safer prescribing of medications in elderly patients [7] so far were mainly based on negative lists such as the Beers list [8] which compiles potentially inappropriate medications (PIMs) and is constantly updated [9]. the base for FORTA-assisted selection of drugs to avoid overtreatment (drug not necessary), undertreatment (condition not or not sufficiently treated by positively labeled drugs) or mistreatment (drugs indicated, but negatively rather than positively labeled drug chosen). Selection is followed by secondary analyses, e.g. regarding contraindications (e.g. allergies), former drug responses, interactions, route of application, duration and dosing (e.g. renal adaptation). This may lead to iterative process optimization. The medication scheme is updated in reflection of clinical effects (e.g. blood pressure) and side effects (e.g. dizziness).

Conclusion: The FORTA approach as an implicit tool should be embedded into the diagnostic and therapeutic workup of elderly patients and can give pivotal hints for the choice of medications; however it should not be seen as an isolated instrument.

Such "negative lists" are easy to apply as they are explicit tools not requiring in-depth knowledge about the patient, but still lack confirmation as to their effectiveness at the clinical endpoint level [10].

In 2008, Gallagher et al. introduced the START (Screening Tool to Alert Doctors to the Right Treatment)/STOPP (Screening Tool of Older Persons' Prescriptions) Criteria [11], and Wehling the FORTA ("Fit fOR The Aged") classification system [12], in English 2009 [13]. Both approaches combine negative and positive labeling of treatments or drugs for the first time. The START criteria target potential errors of omission by pinpointing treatment schemes which may be indicated but not prescribed [14]; the FORTA classification supports the screening for unnecessary, inappropriate or harmful medications and omissions of individual drugs in older patients. As the first classification system it combines negative and positive labelling at the level 
of individual drugs or drug groups and allows for drug optimization across all major therapeutic areas. FORTA is evidence-based and real-life-oriented; as evidence is scarce for elderly patients it has to comprise consensus elements which is a common feature of all listing approaches.

FORTA defines 4 categories of drugs or drug groups:

- Category A (A-bsolutely)= Indispensable drug, clear-cut benefits regarding efficacy-safety ratio proven in elderly patients for a given indication

- Category B (B-eneficial)= Drugs with proven or obvious efficacy in the elderly, but limited extent of effect or safety concerns

- Category C (C-areful)=Drugs with questionable efficacysafety profiles in the elderly, to be avoided or omitted in the presence of too many drugs, lack of benefits or emerging side effects; review and find alternatives, intense monitoring of effects and side effects is necessary

- Category $D(\mathrm{D}$-on't $)=$ Avoid in the elderly, omit almost always, review and find alternatives

The FORTA List classifies over 200 medications used in long-term treatment [15] and most frequently prescribed in older patients, aligned to over 20 main indication groups. It addresses the "elderly", an ill-defined age-group without authorized and unequivocally accepted criteria; yet those patients may be described best by age alone ( $>80$ years), or by age $>65$ years plus relevant diagnoses with impact on prognosis and independent living, in particular dementia. This freely accessible list was derived from an author-based proposal [16], English version [17], by a 2-stage Delphi process finally involving 25 experts from Germany and Austria. Though exact use data are as yet missing, the original FORTA list is increasingly recognized, as indicated by the fact that the $3^{\text {rd }}$ edition of the seminal book (2013) had to be published within 3 years after the first edition appearing in 2010. First results from an interventional pilot trial [18] indicate that clinical endpoints (e.g. falls) are significantly reduced by application of the FORTA list. A larger confirmatory trial (Sponsor: Deutsche Forschungsgemeinschaft WE 1184/15-1) is presently ongoing.

Based on experiences from these trials and the personal experiences of the author, use information on how to apply the FORTA list in daily clinical practice should be described here. This guidance demonstrates that FORTA as an implicit approach which requires in-depth knowledge of the patient has to be sensibly embedded in the complex decision process to find optimal medications in the elderly; it comprises the individual diseases, relevant functional conditions and secondary drug assessments. FORTA should facilitate this process, but is not sufficient as a freestanding instrument.

\section{Development of a Use Algorithm for FORTA $\nabla$}

Implementing the FORTA list requires preparatory steps before it can be used in a meaningful way (step 1). Its application has to be guided by a structured procedure (step 2); the medication scheme derived should be challenged in a critical, integrative review (step 3). Ultimately the clinical outcome of the interventions should be monitored (step 4). These 4 key elements of the use algorithm of FORTA are described in the following chapters and synthesized into an algorithm or flowchart ( $\bullet$ Fig. 1). It is obvious that the complex algorithm cannot be put into practice without proper education, exercising and use of appropriate tools, e.g. for the evaluation of potential adverse drug reactions, drug-drug interaction, food-drug interactions, errors of medications, geriatric assessment and monitoring. As such it requires a stepwise and dedicated implementation.

\section{Step 1: What is required before application of FORTA?}

A thorough medical history, physical exam and knowledge of key lab results need to be compiled to describe the individual to be treated. Key elements for exploration are

1. Relevant diagnoses

2. Severity and current level of control

3. Current treatments including drugs and reported experiences

4. Co-morbidity and functional status with particular focus on geriatric syndromes such as dementia, frailty, activities of daily living (ADSL) and organ function, in particular renal function

It is obvious that diagnoses are the pivotal conditions of treatment; however, in a multimorbid patient the weighing of their contribution to morbidity and prognosis is essential. Thus, the list of diagnoses requires individualization as in many instances (e.g. for reimbursement purposes) irrelevant diagnoses may be included; they need to be identified, mainly by assessment of their symptomatic and prognostic value. Latter is often not prominent given the overall life expectancy of compromised elderly patients. Thus, the list of diagnoses has to be developed into a list of diagnoses with relevance for treatment; this includes the identification of diagnoses which are not amenable to successful treatment (e.g. severe dementia).

The next step addresses the intensity of treatments in reflection of the present disease severity, treatment modalities and treatment goals which need to be individualized. As an example, the treatment goal for hypertension may be $140 \mathrm{mmHg}$ systolic in one patient who is free of orthostatic symptoms, but not in the other demonstrating such symptoms. Thereby, an initial assessment of current over- or undertreatment issues is achieved.

The current modalities of treatment, in particular drug treatment, require intensive history taking from patients, care givers and relatives, study of related documents; yet medication lists obtained are often incomplete or wrong. In the author's practice, just taking drug history may require $20 \mathrm{~min}$, and results may still be wrong thereafter.

The relevant comorbidities and functional conditions as mentioned above may influence therapeutic intensity (go-go/slowgo/no-go concept depending on individual prognosis and life expectancy, quality of life, see below), and safety considerations (dementia and dementing drugs, renally excreted drugs with narrow therapeutic margins as dangerous drugs).

Functional tests (e.g. Mini Mental Status, Tinetti test) may be required to structure and standardize the process at step 1.

This preparatory step should result in a complete picture of relevant diagnoses to be treated according to a patient's individual characteristics. This is often not covered by existing guidelines which are rarely applicable as the underlying evidence does typically not include the elderly patient (for details see [19]).

\section{Step 2: Structured application of the FORTA list or classification}

3 questions regarding drug therapy should be answered for all diagnoses which are considered to be clinically relevant:

1) Are there current treatments which are unnecessary (overtreatment)? 


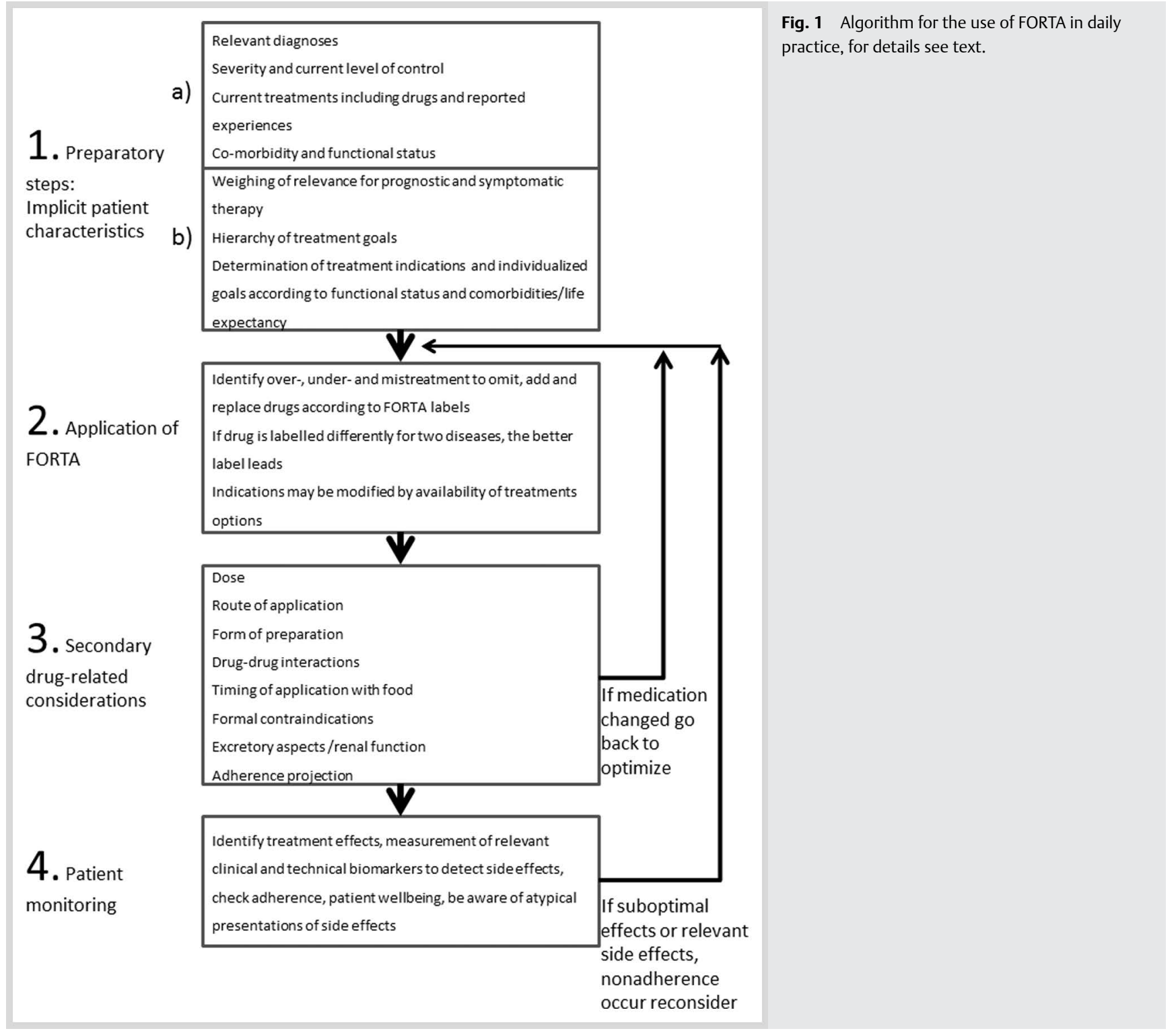

2) Are there diagnoses which require any or at least additional treatment because they are clinically relevant, treatable with positively labelled drugs (mainly FORTA A and B, exceptionally $\mathrm{C}$ ) and so far not sufficiently treated (undertreatment)?

3) Are there diagnoses which are treated to target, but not by the best available drugs according to FORTA (mistreatment, e.g. by $\mathrm{C}$ or even $\mathrm{D}$ drugs though $\mathrm{A}$ or $\mathrm{B}$ drugs are available).

The identification of overtreatment is virtually independent of FORTA and may even concern "good" drugs such as B labeled proton-pump-inhibitors which are often given without indication. It could become FORTA-dependent if a given condition is only treatable by "bad" drugs such as sleep disorders if simple interventions fail and e.g. melatonin does not work. Overtreatment may then simply reflect lack of treatability as defined by FORTA. The main strengths of the FORTA classification, however, relate to under- and mistreatment.

The order in which drugs should be used should be governed by its best label when the same drug has multiple indications and thus maybe different labels. For example, betablockers are labeled $B$ in arterial hypertension, and thus should only be applied if A drugs (ACE-inhibitors/angiotensin-receptor-antagonists and long-acting dihydropyridine calciumantagonists) have been exhausted; in heart failure, they are labeled A, and concomitant arterial hypertension is no longer relevant for their prioritization. An additional strength of FORTA is its cross-therapeutic validity and, thus, potential to assess medication schemes in an integral manner across therapeutic areas. This means that the number of FORTA class $C$ drugs must be limited as by definition they require intense monitoring which needs to remain manageable. As a golden rule no more than 1 or $2 \mathrm{C}$ drugs should be applied even if a larger number may seem indicated by multimorbidity.

The FORTA list does not cover all available drugs and diagnoses; it is rather designed to be practical and useable, and thus only contains 225 items, both for individual drugs or drug groups. Therefore, a given drug may not be listed or included in a group classification, or is not labeled for a rarer indication. 2 ways to cope with these necessary shortcomings are envisioned: the experienced doctor who has experience with the FORTA principle is able to assess the FORTA criteria for the unclassified drug and classify it for his/her own use. Of course, this rating is not validated as opposed to ratings in the list which reflect the scoring by a total of 25 experts. The second approach is more straightforward: if a rated alternative is available this should rather be given than the unrated compound. 
The indication for treatment is the important gatekeeper for a rationalistic treatment as it reflects a patient's individual needs and constitution. For example, primary prevention by a statin (beneficial drug, FORTA A or B) is not indicated if the remaining life expectancy is clearly lower than the time required for clinically relevant endpoint effects to develop. In a former recommendation it was suggested not to use statins in primary prevention if a patient lived healthily up to the $85^{\text {th }}$ birthday [20]. Recommendations on elderly patients with type 2 diabetes mellitus provide staged HbA1c goals depending on the functional and comorbid status, and life expectancy [21]. Many patients for whom an HbA1c of $8.5 \%$ is considered adequate do not require any drug treatment. This concept which was adapted from the "go-go, slow-go and no-go" concept for tumor therapy (for review see, e.g. [22]), also applies to the FORTA based treatments. FORTA does not provide clues about indications though positive labeling of drugs in most instances reflects positive endpoint data in the elderly. Yet, even this fact may not be applicable to the individual to be treated, and drug treatment is not initiated though FORTA A or B drugs are available.

\section{Step 3: Secondary aspects of the medication scheme derived by FORTA}

FORTA does not detail basic parameters of proper drug use such as dose, route of application, form of preparation (e.g. fixed combinations), drug-drug interactions both at the pharmacokinetic and pharmacodynamic level, timing of application with food, formal contraindications such as allergies, excretory aspects (e.g. renal function if renal excretion is predominant) or adherence projection (before adherence can be measured during ongoing treatment). Several of these aspects are covered by the medication appropriateness index (MAI) [23] which is another implicit tool in medication optimization. Though FORTA labels integrate some of those aspects in the overall safety assessment, the individualization (e.g. renal dosing) cannot be included in the simple 4-letter code, and needs to be done in addition. Dosing is a key element for successful drug treatment, and inappropriate dosing in renally impaired patients is a major contributor of unsafe drugs use. Renal dosing should be supported by appropriate tools such as the website "dosing.de", as should be the search for drug-drug-interactions by one of the established electronic databases such IFAB.

Any of those parameters mentioned here may cause failure or adverse effects if not properly addressed. It demonstrates that drug treatment as the most frequently practiced form of treatment in medicine is all but simple, FORTA may just facilitate to establish a structured approach.

If these secondary aspects reveal an unsuitable drug, the FORTA guided search for replacement or omission in the case of insufficient alternatives needs to be repeated as often as necessary (start from step 2).

\section{Step 4: Monitoring of the patient}

Any drug application should be considered as an experiment which may only be successfully done if closely monitored. This means that all assumptions and projections as detailed for the first 3 steps need to be critically evaluated when the patient is exposed to the elected drugs.

Monitoring can be simple (how do you feel?) or very complex if technical measures such as imaging or expensive lab determinations are required. Most important are objective biomarkers to measure the wanted and unwanted effects of a drug. Biomarkers may be blood pressure, pain (both reduced as wanted effect or induced as unwanted effect, e.g. in reflection of gastrointestinal side effect of NSAIDs, for review see [24]), leukocytes for metamizole or more complex measures such as heart function. Renal function should often and routinely be determined, e.g. estimated by the Cockcroft-Gault-formula, as it may be a critical parameter for the excretion of drugs and for side effects of renotoxic drugs (e.g. NSAIDs).

Structured measures to assess side effects (e.g. the Naranjo Score) as well as lists for major expected side effects [25].

The scope of measures employed and the intensity of monitoring must be guided by evidences and extrapolations in relation to the often vulnerable elderly patient. There is no simple rule e.g. for the timing of visits, which goes far beyond the common sense that after initiation of treatment visits should be more often than after longer stable periods of treatment. It is just important to see the patient, ask about specific side effects at appropriate intervals which however may vary and depend on multiple factors. This means that experience and judgment of the physician are the key determinants of this reasoning. Neglect however is common, and "what can go wrong will go wrong" rendering even good drugs into dangerous ones. This is also reflected by the rule not to apply more than one or two $\mathrm{C}$ drugs to the same patient.

The results of monitoring may lead back to the FORTA guided drug selection process for replacement of failed treatment attempts. The individual use experience is more valuable than any projection and should be seen as most precious information to guide the FORTA-based drug selection. No classification can overrule the individual effects and experience. Clinical surveillance is necessary for all FORTA categories, and intense surveillance mandated for $\mathrm{C}$ drugs as an integral characteristic of this category.

\section{Discussion}

$\nabla$

The FORTA system describes an evidence-based classification of medications according to age-appropriateness. Though clinically invalidated so far, it is the first listing approach to both label drugs positively and negatively as deemed necessary. First clinical studies seem to indicate its clinical utility in that endpoint effects were seen e.g. in a reduction of falls and improved Barthel index [18]. It also showed significant improvement of overand undertreatment, but no reduction of average numbers of drugs and polypharmacy. Obviously, modern medications offer valuable opportunities, and multiple medications may well be indicated. The study shows that quality in terms of avoiding both over- and undertreatment may be more important than the number of drugs. In addition, in a small feasibility study [26] quality of medications improves (more A/B, less C/D medications) if FORTA is applied.

It has become evident that a major strength, but also challenge is the fact that applying FORTA requires in-depth knowledge of the patient; the 3 major shortcomings of all therapies (over-, under- and mistreatment) are only accessible if patient details are known. Explicit tools such as the negative lists (e.g. Beers list) [27] can be applied without knowing the patients' details and identify drugs which should be avoided in all elderly patients regardless of their diagnoses and functional status. In contrast, the use of FORTA would not make sense if e.g. A or B labeled drugs would be applied to all geriatric patients. As an 
implicit tool the use of FORTA has to be individualized; it definitely requires a thorough characterization of the patient to find optimal medications for relevant diagnoses. The instructions for the use of FORTA described here have been developed during extensive use in clinical studies and daily clinical practice. The algorithm depicted in 0 Fig. 1 clearly demonstrates the complexity of prescribing in the elderly which has to reflect a multimodal process. This scheme also shows that the very variable characteristics of elderly patients require an intense individualization, in particular as they are typically not covered by common guidelines [19]. Electronic support systems may be helpful to compute essential information which is instantly accessible. It is, however, not imaginable how such systems could replace reasoning and integration of complex medication schemes by clinical judgement though the FORTA instrument should be developed into a more searchable tool (e.g. an "app") than those currently downloadable versions.

FORTA may be seen as a tool to optimize the medication process under those restraints in general practices. So far, it is the only implicit principle which labels drugs and drug groups to allow for a cross-therapeutic assessment. With the START criteria [14] additive prescribing would occur if the patient meets several START criteria. The MAI [23] as another implicit tool adds many of its valuable aspects to the process described here, but does not really rate individual drugs or drug groups in relations to diagnoses in a list.

Finally, it is obvious that in those typical octogenarians with over 3 major diagnoses medication optimization cannot be achieved within the paid consultation time of less than $10 \mathrm{~min}$ in German general practices [28]. Technical measures such as imaging or invasive interventions are generally overpaid, and what is called "thinking and speaking" medicine vastly underpaid. Doctors do not receive any special remuneration for the prescription of drugs.

\section{Limitations}

$\nabla$

The utility of FORTA has still to be proven at the practical level, but the algorithm described here for its use should not only help to implement FORTA, but also to structure medication processes in a more generic way. It thus could be applied to other implicit instruments as well.

The main limitations of the described algorithm for the FORTA approach are

- the lack of experiences for its implementation und utility in daily practice which need to be collected in the ongoing and future studies.

- The inter-rater reliability has yet to be determined; preliminary, unpublished observations for the re-test reliability ranged from 0.727 to 1.0 for the different FORTA classes.

- The impact of the FORTA list on medication quality and clinical endpoints and the feasibility of its implementation into clinical practice have to be confirmed in prospective interventional studies which are currently performed (see introduction).

- Further evaluation of reliability between health professionals practicing in different centers is warranted to determine if FORTA instructions and list are generalizable.

\section{Summary}

$\nabla$

A novel listing approach (the FORTA list) to improve medication in the elderly is discussed in regard of the complex medication process in the elderly.

FORTA labels range from A (indispensable), B (beneficial), C (questionable) to $\mathrm{D}$ (avoid).

As implicit tool it is only applicable if medical details of the patient are known.

It should be smartly embedded into the diagnostic and therapeutic workup of elderly patients.

Early validation of FORTA indicates reduced fall risk and improved medication quality.

\section{Acknowledgements}

$\nabla$

Christine Schummer is gratefully acknowledged for her secretarial support.

\section{Conflicts of Interest}

$\nabla$

The author was employed by AstraZeneca R\&D, Mölndal, as director of discovery medicine (=translational medicine) from 2003-2006, while on sabbatical leave from his professorship at the University of Heidelberg. Since returning to this position in January 2007, he has received lecturing and consulting fees from Sanofi-Aventis, Bayer, Boehringer-Ingelheim, Novartis, Takeda, Roche, Pfizer, Bristol-Myers, Daichii-Sankyo, Lilly, Otsuka, NovoNordisk, Shire and LEO Pharma.

\section{References}

1 Kaufman DW, Kelly JP, Rosenberg L et al. Recent patterns of medication use in the ambulatory adult population of the United States: the Slone Survey. JAMA 2002; 287: 337-344

2 Mangoni AA. Jackson SHD. Age-related changes in pharmacokinetics and pharmacodynamics: basic principles and practical applications. Br J Clin Pharmacol 2003; 57: 6-14

3 Edwards IR, Aronson JK. Adverse drug reactions: definitions, diagnosis, and management. Lancet 2000; 356: 1255-1259

4 Rochon PA, Gurwitz JH. Optimising drug treatment for elderly people: the prescribing cascade. Br Med J 1997; 315: 1096-1099

5 Chyka PA. How many deaths occur annually from adverse drug reactions in the United States? Am J Med 2000; 109: 122-130

6 Lazarou J, Pomeranz BH, Corey PN. Incidence of adverse drug reactions in hospitalized patients: a meta-analysis of prospective studies. JAMA 1998; 279: 1200-1205

7 Levy HB, Marcus EL, Christen C. Beyond the Beers Criteria: a comparative overview of explicit criteria. Ann Pharmacother 2010; 44: 1968-1975

8 Beers $M H$. Explicit criteria for determining potentially inappropriate medication use by the elderly. An Update. Arch Intern Med 1997; 157: $1531-1536$

9 Fick DM, Semla TP. American Geriatrics Society Beers Criteria: new year, new criteria, new perspective. J Am Geriatr Soc 2012; 60: 614615

10 Page RL, Ruscin JM. The risk of adverse drug events and hospitalrelated morbidity and mortality among older adults with potentially inappropriate medication use. Am J Geriatr Pharmacother 2006; 4: 297-305

11 Gallagher P, Ryan C, Byrne $S$ et al. STOPP (Screening Tool of Older Person's Prescriptions) and START (Screening Tool to Alert Doctors to Right Treatment). Consensus validation. Int J. Clin Pharmacol Ther 2008; 46: 72-83

12 Wehling M. Drug therapy in the elderly: too much or too little, what to do? A new assessment system: fit for the aged FORTA. Dtsch Med Wochenschr 2008; 133: 2289-2291 (in German) 
13 Wehling M. Multimorbidity and polypharmacy: how to reduce the harmful drug load and yet add needed drugs in the elderly? Proposal of a new drug classification: fit for the aged. J Am Geriatr Soc 2009; 57: $560-561$

14 Barry PJ, Gallagher PRyan et al. START-an evidence-based screening tool to detect prescribing omissions in elderly patients. Age Ageing 2007; 36: 632-638

15 Kuhn-Thiel AM, Weiß C, Wehling M. The FORTA authors/expert panel members. Consensus validation of the FORTA (Fit fOR The Aged) list: a clinical tool for increasing the appropriateness of pharmacotherapy in the elderly. Drugs Aging 2014; 31: 131-140

16 Wehling $M$, Burkhardt $H$. Arzneitherapie für Ältere. $2^{\text {nd }}$ (ed.). Heidelberg: Springer, 2011 (in German)

17 Wehling M. Drug therapy for the elderly. Vienna: Springer Publishers, 2013

18 Michalek C, Wehling $M$, Schlitzer J et al. Effects of "Fit fOR The Aged" (FORTA) on pharmacotherapy and clinical endpoints-a pilot randomized controlled study. Eur J Clin Pharmacol 2014; 70: 1261-1267

19 Wehling $M$. Guideline-driven polypharmacy in elderly, multimorbid patients is basically flawed: there are almost no guidelines for these patients. J Am Geriatr Soc 2011; 59: 376-377

20 Doser S, Marz W, Reinecke MF et al. Recommendations for statin therapy in the elderly. Internist 2004; 45: 1053-1062
21 Sue Kirkman M, Briscoe VJ, Clark $N$ et al. Diabetes in older adults: a consensus report. J Am Geriatr Soc. 2012; 60: 2342-2356

22 Balducci L, Extermann M. Management of cancer in the older person: a practical approach. Oncologist 2000; 5: 224-237

23 Hanlon JT, Schmader KE, Samsa GP et al. A method for assessing drug therapy appropriateness. J Clin Epidemiol 1992; 45: 1045-1051

24 Conaghan PG. A turbulent decade for NSAIDs: update on current concepts of classification, epidemiology, comparative efficacy, and toxicity. Rheumatol Int 2012; 32: 1491-1502

25 Trivalle C, Cartier T, Verny $C$ et al.IMEPAG GROUP. Identifying and preventing adverse drug events in elderly hospitalised patients: a randomised trial of a program to reduce adverse drug effects. J Nutr Health Aging 2010; 14: 57-61

26 Frohnhofen H, Michalek C, Wehling M. Assessment of drug treatment in geriatrics with the new FORTA criteria - Preliminary clinical experience. Dtsch Med Wochenschr 2011; 136: 1417-1421 (in German)

27 American Geriatrics Society 2012 Beers Criteria Update Expert Panel. American Geriatrics Society updated Beers Criteria for potentially inappropriate medication use in older adults. J Am Geriatr Soc 2012; 60: 616-631

28 Schoen C, Osborn R, Huynh PT et al. On the front lines of care: primary care doctors' office systems, experiences, and views in seven countries. Health Aff (Millwood) 2005; 25: w555-w571 